\title{
THE ROLE OF TEACHING IN ITERATED LANGUAGE TRANSMISSION
}

\author{
Vera Kempe ${ }^{* 1}$, Kamil Cichon ${ }^{1}$, Monica Tamariz ${ }^{2}$ and Nicolas Gauvrit ${ }^{3}$ \\ *Corresponding Author: v.kempe@abertay.ac.uk \\ ${ }^{1}$ Division of Psychology, Abertay University, Dundee, Scotland, UK \\ ${ }^{2}$ Department of Psychology, Heriot Watt University, Edinburgh, Scotland, UK \\ ${ }^{3}$ Université Paris 8 \& École Pratique des Hautes Études, Paris, France
}

Iterated language learning studies have shown that constraints on learning and communication drive emergence of linguistic structure (e.g. Kirby, Tamariz, Cornish \& Smith; 2015). However, language is typically passed on by experts who select and modify language input in ways that facilitates learning by novices. Research on first language acquisition has shown that the kind of input enhancement that characterises child-directed speech is beneficial for language learning (e.g. Eaves, Feldman, Griffiths \& Shafto, 2016). This suggests that in addition to its other functions such as expressing positive affect, promoting emotional bonding and regulating infant arousal (Singh, Morgan \& Best, 2002; Uther, Knoll \& Burnham, 2007), this speech register can be considered a form of teaching (Kline, 2015). While the adaptive value of teaching for the transmission of complex cultural traits, and the role of language as a medium of teaching, have recently received increased attention (e.g. Csibra \& Gergely, 2009; Laland, 2017), detailed explorations of the effect of teaching on the transmission of language itself are lacking. Here we use iterated teaching of a simple signalling system to explore how language change that emerges during transmission is affected by experts' intention to teach the language to novices.

We compared six transmission chains, of ten generations each, in which a participant's language output after learning was saved and then transmitted to the next participant by the experimenter (simple transmission condition) to six transmission chains in which the participants themselves were asked to teach the next participant in the chain (teaching condition). Participants learned an "alien" language consisting of binary auditory sequences of high and low tones produced using two programmable buzzers. At the outset, eight randomly 
generated 6 or 8-bit binary auditory sequences were associated with meanings representing kiki- and bouba-type objects differing in size (large vs. small), shape (spiky vs. round) and brightness (dark vs. light) while still retaining individuating features. Signal-meaning pairs were presented twice in incremental fashion. In the simple transmission condition, only six signalmeaning pairs were presented and homophones were removed, to prevent languages from degenerating into ambiguous systems. In the teaching conditions, participants saw all eight pairs and were asked to refrain from speaking while demonstrating the language to the next learner.

The results showed that while in the simple transmission condition individual signals increased in length and languages degenerated slightly despite the homophone filter, in the teaching condition signals retained their length and languages remained expressive by retaining unique signals for the meanings. Similarity of signals within languages increased in both conditions suggesting that learners adopted a set of constraints governing signal production. Algorithmic complexity of individual signals did not decrease and iconicity and compositional structure did not emerge in either condition, presumably due to lack of communicative pressure. Crucially, however, transmission fidelity improved only in the simple transmission condition but not in the teaching condition suggesting that teachers introduced many innovations. We analysed to what extent these teacher innovations were beneficial for learners, and found that while signals did not become easier for learners to reproduce over the course of transmission, they became easier to repeat immediately, both on the first and second attempts. Intriguingly, further analyses revealed that most teacher innovations were triggered by learner repetition errors, and that when learners had erred on their first repetition attempt, they were more likely to correctly repeat the signal on the second attempt the more the teachers' second signals were modified to resemble their first erroneous repetitions. Thus, learner repetition ability was aided by teacher accommodation to learner responses.

These findings suggest that iterated language teaching can affect some functional aspects of language such as expressivity and ease of repetition. We suggest that such teaching effects can arise through two processes: On the one hand, teaching involves spontaneous modifications, i.e. input pre-selection and enhancement, based on teachers' assumptions about learners' knowledge. On the other hand, teaching accommodates direct learner feedback as teachers' productions come to resemble learner responses during episodes of didactic and communicative interaction. Further research should explore the differential effects of these component processes of teaching during language transmission. 


\section{References}

Csibra, G. \& Gergely, G. (2009). Natural pedagogy. Trends in Cognitive Science, 13, 148-153.

Eaves Jr, B. S., Feldman, N. H., Griffiths, T. L., \& Shafto, P. (2016). Infantdirected speech is consistent with teaching. Psychological Review, 123(6), 758.

Kirby, S., Tamariz, M., Cornish, H., \& Smith, K. (2015). Compression and communication in the cultural evolution of linguistic structure. Cognition, $141,87-102$.

Kline, M. A. (2015). How to learn about teaching: An evolutionary framework for the study of teaching behaviour in humans and other animals. Behavioural and Brain Sciences, 38, E31.

Laland, K. N. (2017). The origins of language in teaching. Psychonomic Bulletin \& Review, 24(1), 225-231.

Singh, L., Morgan, J. L., \& Best, C. T. (2002). Infants' listening preferences: Baby talk or happy talk? Infancy, 3(3), 365-394.

Uther, M., Knoll, M., \& Burnham, D. (2007, January). Do you speak E-NG-L-ISH? A comparison of foreigner- and infant-directed speech. Speech Communication, 49(1), 2-7. 